\title{
Utilization Review of Antihypertensive Agents at an Outpatient Pharmacy Setting of a Private Hospital, Selangor, Malaysia
}

Elammathi Ravinthiran ${ }^{1}$, Ching Siang Tan1*, Shashidharan Menon ${ }^{1}$, H. Jaasminerjiit Kaur ${ }^{1}$, Anandarajagopal Kalusalingam ${ }^{1}$, Abdullah Khan ${ }^{1}$, Mohamad Mansor Manan ${ }^{2}$, Khang Wen Goh ${ }^{3}$, Mei Jun Loy ${ }^{4}$, Long Chiau Ming ${ }^{5 *}$

${ }^{1}$ School of Pharmacy, KPJ Healthcare University College, Nilai, Malaysia

${ }^{2}$ Faculty of Pharmacy, Universiti Teknologi MARA, Puncak Alam, Selangor, Malaysia

${ }^{3}$ Faculty of Science and Technology, Quest International University Perak, Ipoh, Perak, Malaysia

${ }^{4}$ Faculty of Engineering, Universiti Teknologi Malaysia, Skudai, Johor, Malaysia

${ }^{5}$ PAPRSB Institute of Health Sciences, Universiti Brunei Darussalam, Gadong, Brunei

\section{Darussalam Abstract:}

Introduction: Drug utilization review of antihypertensive agents is regularly conducted to monitor the prescribing trends. Objectives: The objectives of this study were to describe the drug utilization pattern of antihypertensive agents in the outpa-tient setting of a private hospital and to determine the defined daily dose (DDD) used by the private hospital in comparison to WHO DDD.

Method: This is a retrospective study using pharmacy dispensing record. Inclusion criteria were prescriptions issued in 2017, patients more than 18 years old. The DDD was calculated for each anti-hypertensive drug prescribed which was then compared with WHO DDD. Medicine prices were also analysed.

Results: From the total outpatient prescriptions in 2017, 200 prescriptions were chosen randomly using SPSS software. Based on the prescriptions analysed, the mean number of drugs prescribed per prescription was 3.89 (SD: 2.37). Beta blockers were the most prescribed group of anti-hypertensives (37\%). Bisoprolol 5mg (9\%) and furosemide 40mg (8.7\%) were those drugs given to the most patients. Out of 330 drugs prescribed, $76 \%$ of them were non-fixed dose combination formula-tions. Of note, DDD of the $14 \%$ and $39 \%$ of the anti-hypertensive drugs analysed complied with and of doubled of the WHO DDD, respectively. Out of 330 anti-hypertensive drug products prescribed, $76 \%$ of them were non-FDC drugs.

Conclusion: Beta blockers are still commonly prescribed antihypertensive agents. Compared with similar studies from the last five years, prescribing of newer anti-hypertensive drugs is increased, as evidenced by certain FDC products doubled the DDD value recommended by WHO.

Keywords: Pharmacoepidemiology; cardiovascular diseases; prescribing pattern; Defined Daily Dose

Received: $2^{\text {nd }}$ August 2020

Accepted: $3^{\text {rd }}$ September 2020

Published Online: $18^{\text {th }}$ September 2020
Correspondence: Ching Siang Tan, School of Pharmacy, KPJ Healthcare University College, Nilai, Malaysia; tcsiang@kpjuc. edu.my. Long Chiau Ming, PAPRSB Institute of Health Sciences, Universiti Brunei Darussalam, Gadong, Brunei Darussalam; long. ming@ubd.edu.bn.

Citation: Ravinthiran E, Tan CS, Menon S, et al. Utilization Review of Antihypertensive Agents at an Outpatient Pharmacy Setting of a Private Hospital, Selangor, Malaysia. Prog Drug Discov Biomed Sci 2020; 3(1): a0000111. https://doi.org/10.3687/pddbs.a0000111

\section{Introduction}

Hypertension is referred as a condition of long-term elevated Blood Pressure (BP) that may lead to unwanted morbidities and mortalities. From medical theory, BP is the product of cardiac output and systemic vascular resistance. Patients with arterial hypertension may have an increased cardiac output, an increase in systemic vascular resistance, or both ${ }^{[1]}$. Apart from that, renin-angiotensin system is also responsible to control $\mathrm{BP}^{[2]}$.
According to a recent study, hypertension is one of the most common health related problems which has more potential risk of contribution to initiate and progress major cardiovascular diseases, kidney failure and stroke ${ }^{[3,4]}$. Hypertension is the second leading cause of chronic renal disease. The estimation shows that there are almost billion adults having hypertension and will be increased to 1.56 billion by 2025 worldwide ${ }^{[5]}$ predicting an increase of sixty percent from the year of $2000^{[5]}$. 
Management of hypertension has two approaches which emphasises on lifestyle modification and pharmacological therapy.

Lifestyle measures such as diet, weight management and exercises alone only comply to pre-hypertensive patients or as supportive therapy. National Institute of Health, USA conducted a programme "Dietary Approaches to Stop Hypertension (DASH)" to reduce systolic and diastolic BP value of 11.6 and $5.3 \mathrm{mmHg}$ in hypertensive individuals ${ }^{[6]}$. Besides, utilization of various classes of antihypertensive agents that act by different mechanisms to regulate the elevated BP. According to 2017 Guideline for the Prevention, Detection, Evaluation, and Management of High BP in Adults, the classification of hypertension has been changed, where the reading of elevated $\mathrm{BP}$ is said to be $120-129 /<80 \mathrm{mmHg}^{[7]}$.

Since the year 2000, the international report shows increasing prevalence of hypertension in low- and middle income nations whereas in high-income countries is stable and reducing ${ }^{[8,9]}$. In 2000s, $26.4 \%$ of the global adult population had hypertension and the statistics has increased by almost $5 \%$ within ten years ${ }^{[9]}$. One in three or 6.1 million Malaysians have hypertension based on results from National Health and Morbidity Survey 2015 ${ }^{[10]}$. Meanwhile, the report on Non-Communicable Diseases, Risk Factors \& Other Health Problems by Ministry of Health Malaysia identified the Perak state of Malaysia having the highest prevalence of hypertension (17.3\%) followed by Sarawak (16.5\%) and Negeri Sembilan $(16.1 \%)$ whereas the lowest is in Putrajaya

$(10 \%)^{[11]}$.

The drugs prescribed for hypertension varied based on patient's condition like presence of comorbidities. This type of patient's condition meets the requirement to undergo combination therapy to achieve targeted BP lev-el. The comorbid condition is highly prevalent in older adults consequently each comorbidity increases the total annual medical expenditure ${ }^{[12,13]}$. On the other hand, the cost of antihypertensives used to be the barrier in effective treatment which might influence the prescribing pat-tern and patient compliance ${ }^{[14]}$. The impact of antihypertensive drugs on healthcare cost is very huge. Malaysian Statistics on Medicines announced that RM508 million was spent on antihypertensive medications for public and private healthcare divisions in 2008 compared to RM457 million in $2007^{[15]}$. Conversely, American Heart Association (ASA) found that cardiac medications costs $45 \%$ of total direct $\operatorname{cost}^{[16]}$. Thus, it is imperative to monitor the trend of anti-hypertensives prescribed to determine its impact of cost burden in managed health care system.

Several clinical practice guidelines on management of hypertension have been developed worldwide as a standard reference for healthcare professionals. However, most of the prescribers still practicing their own prescribing pattern based on their experience, rather on evidence-based practices.

Monotherapy or combination therapy of diuretics, betablocker, calcium channel blocker (CCB), angiotensin converting enzyme inhibitors (ACEI), angiotensin rece- ptor blockers (ARB), direct renin inhibitors (DRI), and other drugs included in Malaysian Clinical Practice Guidelines on management of hypertension that could be used to treat elevated BP. In fact, hypertension comes with comorbidities which results in taking more medications thereby increasing the risk of adverse drug reactions and contraindications among them. In order to avoid those issues, following clinical guidelines is crucial.

Nevertheless, some preliminary observation in certain hospitals confirmed that the issue of polypharmacy is increasing and choices of drug therapy do not comply fully with the guidelines. Antihypertensive prescribing patterns need to be examined to determine whether the doctors' adhere to National Clinical Practice Guidelines. Additionally, improper prescribing of antihypertensive is an example of irrational use of drug which may lead to unwanted risk. This project was aimed to describe the utilization patterns of anti-hypertensive agents with or without comorbidities in outpatient department at the selected hospital.

Drug utilization research is needed to facilitate rational use of medicines by describing how the patterns of drugs are used. This study compares the drug use pat-terns whether it complies with the current guidelines or not. Based on this, recommendations are given to the prescribers. It helps to assess whether the suggested plans or interventions could improve the drug usage and outcomes ${ }^{[17]}$. To sum up, drug utilization study also plays a role as a pharmaceutical tool to implement evidence based medicine ${ }^{[18]}$.

\section{Methodology}

\section{Study Design}

This was a retrospective study on the utilization of antihypertensive drugs conducted in outpatient department of a private hospital in Klang Valley area. This study covered a period of 12 months starting from January 2017 till December 2017. The relevant data were collected from hospital's electronic records using KPJ Clinical Information System (KCIS) and Hospital Information Technology System (HITS) and also from poison book.

KCIS is a system that caters to the clinical activity needs of the hospitals to help seamless communication among healthcare professionals. KCIS system consists of patient clinical information, e-pharmacy, e-ordering and clinical reports. Whereas, HITS is an integrated system that has complete range of patient's services from registration to billing. HITS also have functionalities that cover financial and material management, making it truly reliable and robust system.

Data collection from these records was helpful to continue this study and accurate data were provided. Inclusion and exclusion criteria were applied to select the appropriate drug utilization data (KPJ Annual Report $)^{[19]}$.

\section{Sample Size and Sampling Method}

Outpatient prescriptions from the selected hospital were used for analysis. The prescriptions were selected from outpatient poison book. Prescriptions containing anti- 
hypertensive drugs were selected randomly which was around 30 per month. Then KCIS system was accessed to collect more data that were not present in poison book like duration of treatment and frequency of drug pre-scribed. In order to collect data regarding cost of drugs, HITS were assessed. Once the data were gathered, it was chosen by simple random sampling method to select the sample size needed. The sample size was calculated based on the Yamane formula. The estimated sample size based on this calculation is 138 samples. To allow for loss by incomplete data, twenty percentage which is about 28 samples was added. Inclusion and exclusion criteria were applied so that the targeted population for this study would be assessed and suitable participants could be chosen to meet the aim of this study. Implying wrong sampling technique would have a risk of misinterpretation of data and biased results ${ }^{[20] \text {. }}$

\section{Prescription and Patient Record Selection \\ Criteria}

Prescription and patient record were screened using the following ${ }^{[21]}$

\section{Inclusion criteria:}

- Female or male who is currently on antihypertensive medications either only taking antihypertensive drugs or together with other drugs

- $\quad$ Age is more than 18 years old

- $\quad$ Prescription is issued between January 2017 and December 2017

Exclusion criteria:

- Those with incomplete data

- Prescription from different hospital

- Pregnant patients

\section{Statistical Analysis}

Demographic data collected and prescribing record of each patient were analysed descriptively by using the Statistical Package for Social Science (SPSS) program version 22.0. The results were expressed in terms of mean and standard deviation. Data of descriptive statistics was then presented in frequencies and percentages. These collected data were presented in graphs, tables and charts.

\section{Outcome Parameters}

In this study, the data were in DDD per 1,000 inhabitants per day. The DDD results were then compared with the current WHO classification of ATC/DDD for the DDD analysis, obtained from the ATC/DD Index 2018, which are searchable through the website https://www.whocc. no/atc_ddd_index/.

DDD were obtained by using the following formula for single drugs:
A. Rates per residents per day
Rates/residents/day $=$ DDD rates/days per month/ residents X 1000
B. Rates per user per day

Rates/user/day = DDD rates/days per month/users

C. Intermediate rate

Intermediate rates $=$ Total duration $($ days $) /$ Total users

\section{Fixed dose combination drugs:}

DDD assignment for fixed combinations differs from that for single ingredient products. The DDD for fixed combinations, such as ACEIs or ARBs with diuretics, do not depend on the dose rate of the single ingredients, but are based on the average number of dosing intervals per day. For example, one tablet is the DDD for combinations given once daily, two tablets are the DDD for combinations given twice daily etc.

\section{Ethical Considerations}

Ethical issues such as confidentiality and informed consent were considered in this study. This has been made compulsory to protect the rights and privacy of each patient ${ }^{[22]}$. This included anonymized patient and seeking relevant permission from the ethical authority ${ }^{[23]}$. This application for research (Ref No. KPJUC/RMC/BPH/EC/2017/103) had been approved by Research Ethic Committee of KPJ Healthcare University College, Nilai, Malaysia.

\section{Results}

The prescription data obtained from the respective hospital setting were selected based on the criteria such as the patient age must be more than 18 years old. Data that met all the inclusion criteria were critically analysed. From the total outpatient prescriptions in 2017, 300 prescriptions were found to match the criteria based on the study's requirement. However, due to some incomplete data, such as no prescribers' details stated, no frequency and duration of prescribed has written in the system only 224 prescriptions were selected and filtered out, finally 200 prescriptions were chosen randomly by using SPSS software. More than calculated sample size was considered into this study to get more accurate results.

\section{Sociodemographic Characteristics of Patients}

The 200-prescription data were presented based on demo-graphic characteristics of patients as gender, ethnicity and their age categorization.

\section{Demographic}

A total of 200 prescriptions with patient record fulfilled the inclusion criteria were analysed in this study. Among the sample that were involved in this study, $52.50 \%$ were male while $47.50 \%$ were female. Highest number of patients being Malays with 55\%, followed by Chinese 34\% and the least is Indians and other races with equal percent-ages of $5.5 \%$.

Most patients that found to have hypertension are those who are above 55 years old which is around $52.5 \%$ followed by middle aged adults who is in between age of 36 to 55 with $43 \%$. Only $4.5 \%$ of the young adults found to have hypertension in this study. The mean age is $\mathbf{5 7 . 8 2}$ years old. Table 1 summarizes the demographic data. 
Table 1. Summary of demographic data

\begin{tabular}{|c|c|c|c|c|c|}
\hline \multirow{3}{*}{ Age } & \multirow{3}{*}{ Ethnicity } & \multicolumn{4}{|c|}{ Patient gender } \\
\hline & & \multicolumn{2}{|c|}{ Male } & \multicolumn{2}{|c|}{ Female } \\
\hline & & $\mathrm{n}$ & $\%$ & $\mathrm{n}$ & $\%$ \\
\hline \multirow[t]{5}{*}{$18-35$} & Malay & 1 & 0.5 & 2 & 1 \\
\hline & Chinese & 2 & 1 & 3 & 1.5 \\
\hline & Indian & $\mathbf{0}$ & 0 & $\mathbf{0}$ & 0 \\
\hline & Others & 1 & 0.5 & $\mathbf{0}$ & 0 \\
\hline & Total & 4 & 2 & 5 & 2.5 \\
\hline \multirow[t]{5}{*}{$36-55$} & Malay & 32 & 16 & 21 & 10.5 \\
\hline & Chinese & 10 & 5 & 12 & 6 \\
\hline & Indian & 3 & 1.5 & 1 & 0.5 \\
\hline & Others & 4 & 2 & 3 & 1.5 \\
\hline & Total & 49 & 24.5 & 37 & 18.5 \\
\hline \multirow[t]{5}{*}{$>55$} & Malay & 28 & 14 & 26 & 13 \\
\hline & Chinese & 21 & 10.5 & 20 & 10 \\
\hline & Indian & 2 & 1 & 5 & 2.5 \\
\hline & Others & 1 & 0.5 & 2 & 1 \\
\hline & Total & 52 & 26 & 53 & 26.5 \\
\hline Mean & \multicolumn{5}{|c|}{57.81} \\
\hline
\end{tabular}

\section{Prescription analysis}

Total number of drugs per prescription

Numbers of drugs prescribed for every hypertensive patient among the 200 samples were analysed. Those results were presented below based on categorized age groups; young adults (18-35 years old), middle-aged adults (3655 years old) and older adults (more than 55 years old).

In this study total number of drugs per prescriptions varied according to age groups. The mean number of drugs prescribed per prescription was 3.89 , therefore no poly pharmacy obtained. Almost $69.5 \%(n=139)$ of patients took less than 5 drugs and $30.5 \%$ ( $n=61$ ) took five or more than five drugs per prescription. The number of drugs per prescription is seen to increase as increasing age.

Table 2 shows the number and percentage of drugs per prescription sorted according to ethnicity and age group. Among the study population, the highest percentage is shown for prescriptions with more than 5 drugs taken by older aged patients ( $>55$ years old) that is accounted for $32 \%(\mathrm{n}=39)$ followed by $24 \%$ of older adults take less than 5 drugs per prescription in this study. While the low-est percentage says only $1 \%$ of young adults are taking five more than five prescriptions drugs.

Table 2. Number and percentage of drugs per prescription sort by ethnicity relating to their age group

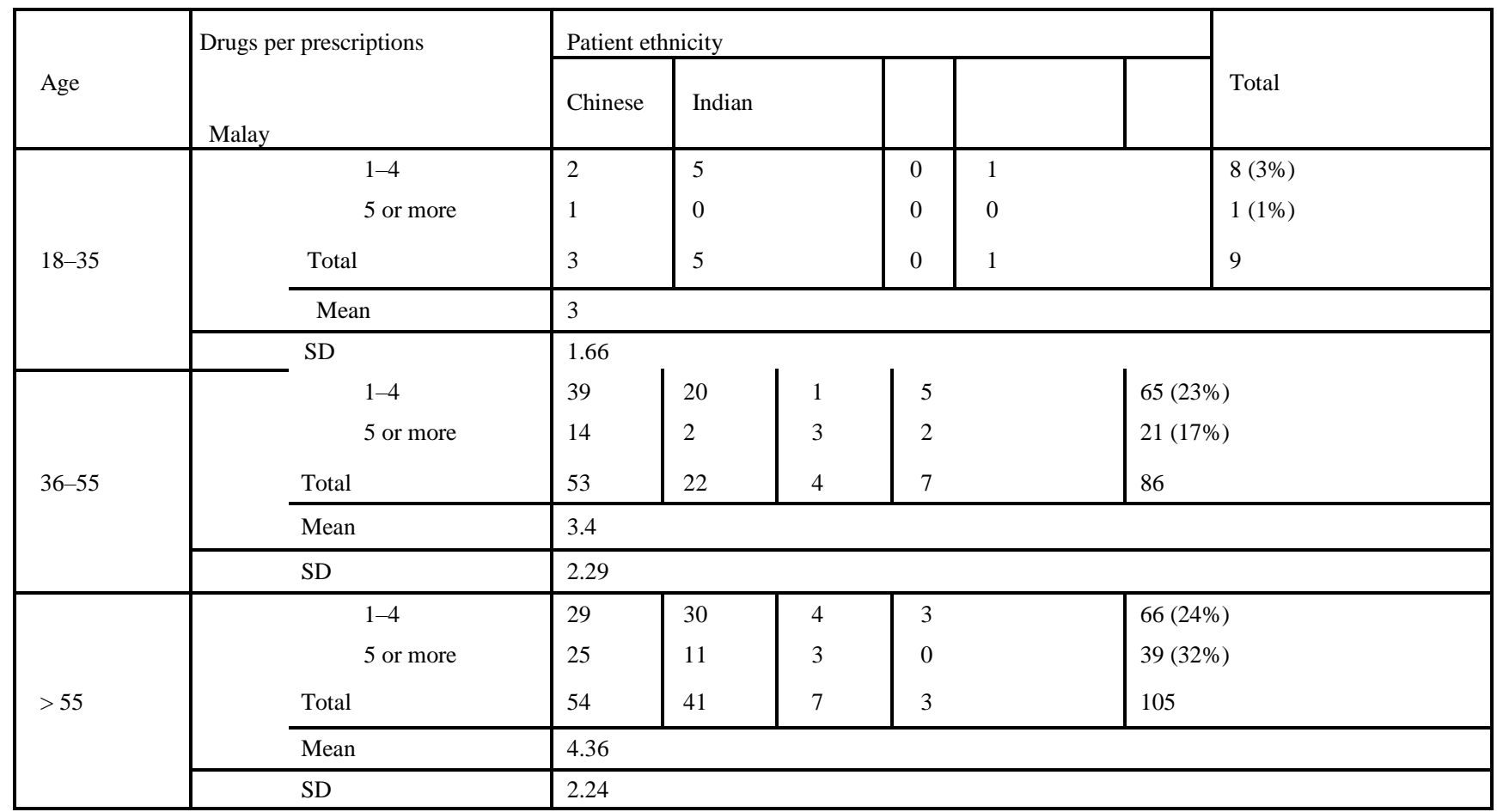


Total number of antihypertensive drugs prescribed per prescription

Figure 1 shows the percentage of anti-hypertensive drugs prescribed per patient. The minimum amount of drug prescribed is 1 and maximum is 6 . The mean number of anti-hypertensive drug per prescription is 2.12 .
Among the age group, $28.6 \%$ of old aged adults received one drug per prescriptions meanwhile $44.4 \%$ of young adults received 1 drug per prescription. A total of $2 \%$ from the older adults received more than 5 type of antihypertensive drugs per prescription. From the age 18-55 years old, none of them received more than 4 drugs per prescription.

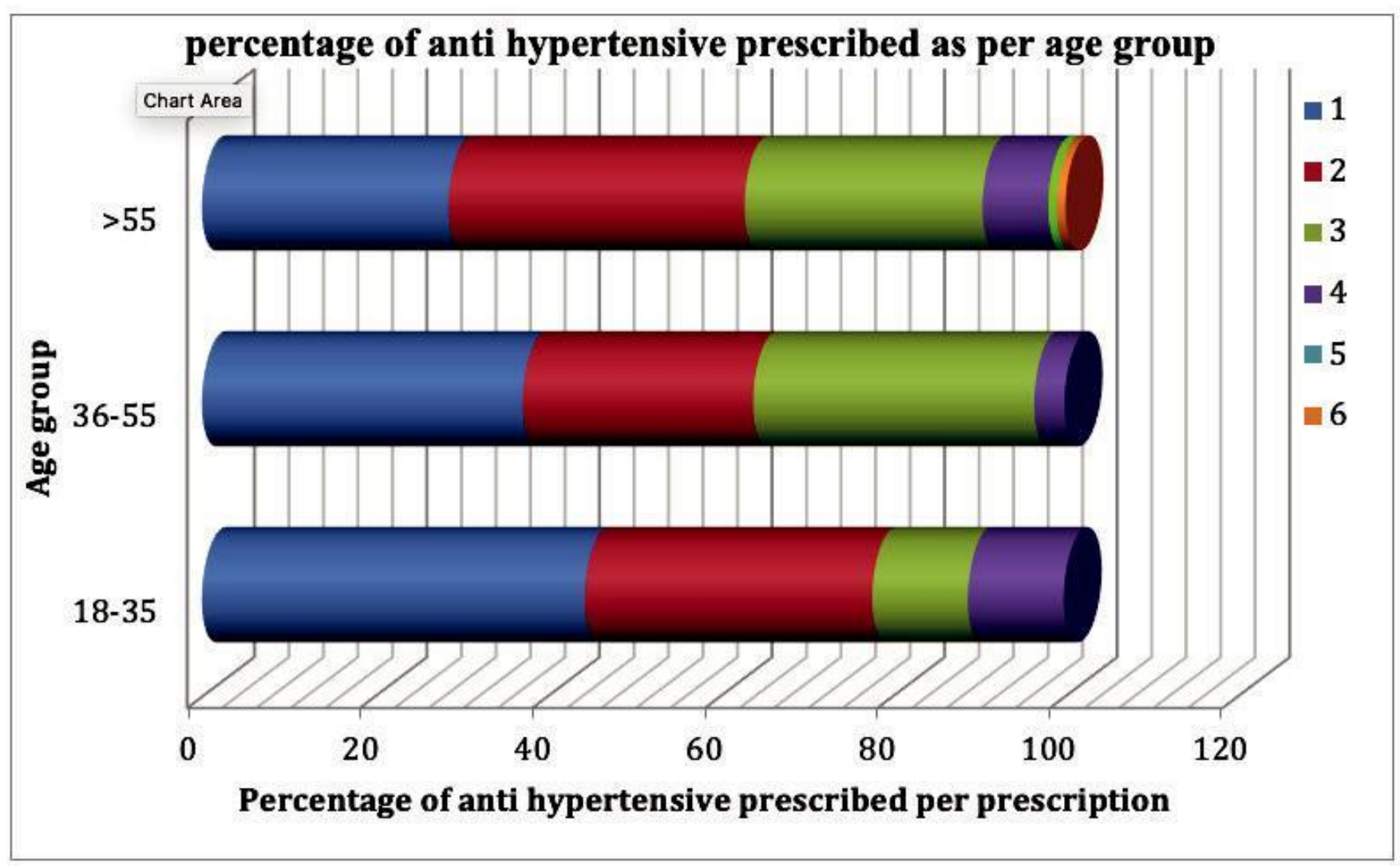

Figure 1. Percentage of antihypertensive drugs prescribed per prescription as per age

\section{Drug pattern and utilization}

Pattern of drugs prescribed based on classes

In this study number and type of antihypertensive drugs prescribed varied according to prescriber's choice and patient's requirement of therapy. Among the 200 prescriptions screened, total number of 330 drugs were prescribed from 11 classes of anti-hypertensive including the fixed dose combination (FDC) drugs. The mean number of drugs prescribed per prescriptions was 1.61.

Out of 330 oral drugs that were prescribed to 200 patients, the most frequently prescribed single anti-hypertensive medication in the corresponding hospital setting were from beta blockers (BB) accounting for $37 \%$ followed by Diuretics $23 \%$ and the lowest prescribed were
ACEi 9\%

Bisoprolol 5mg (9\%) and Furosemide 40mg (8.7\%) were the highest number of drugs prescribed among all classes of antihypertensive drugs in this study. The lowest number of drugs prescribed was Nifedipine $20 \mathrm{mg} \& 60 \mathrm{mg}(0.8 \%)$.

In combination drugs, the highly used class was CCB + ARB (29.5\%) while the lowest was ACEi + Diuretics (4\%). Amlodipine $5 \mathrm{mg}+$ valsartan $80 \mathrm{mg}$ and telmisartan $80 \mathrm{mg}+$ HCTZ $12.5 \mathrm{mg}$ were highly used drugs. Fixed dose combination drugs $(24 \%)$ were used very less compared to single dose drugs (76\%). Among 32 types of drugs prescribed, only $3 \%$ of drugs were prescribed through generic names, whereas, $93 \%$ of drugs were known with their respective brand names. Summary of all drugs prescribed and its frequency were showed in Figure 2. 


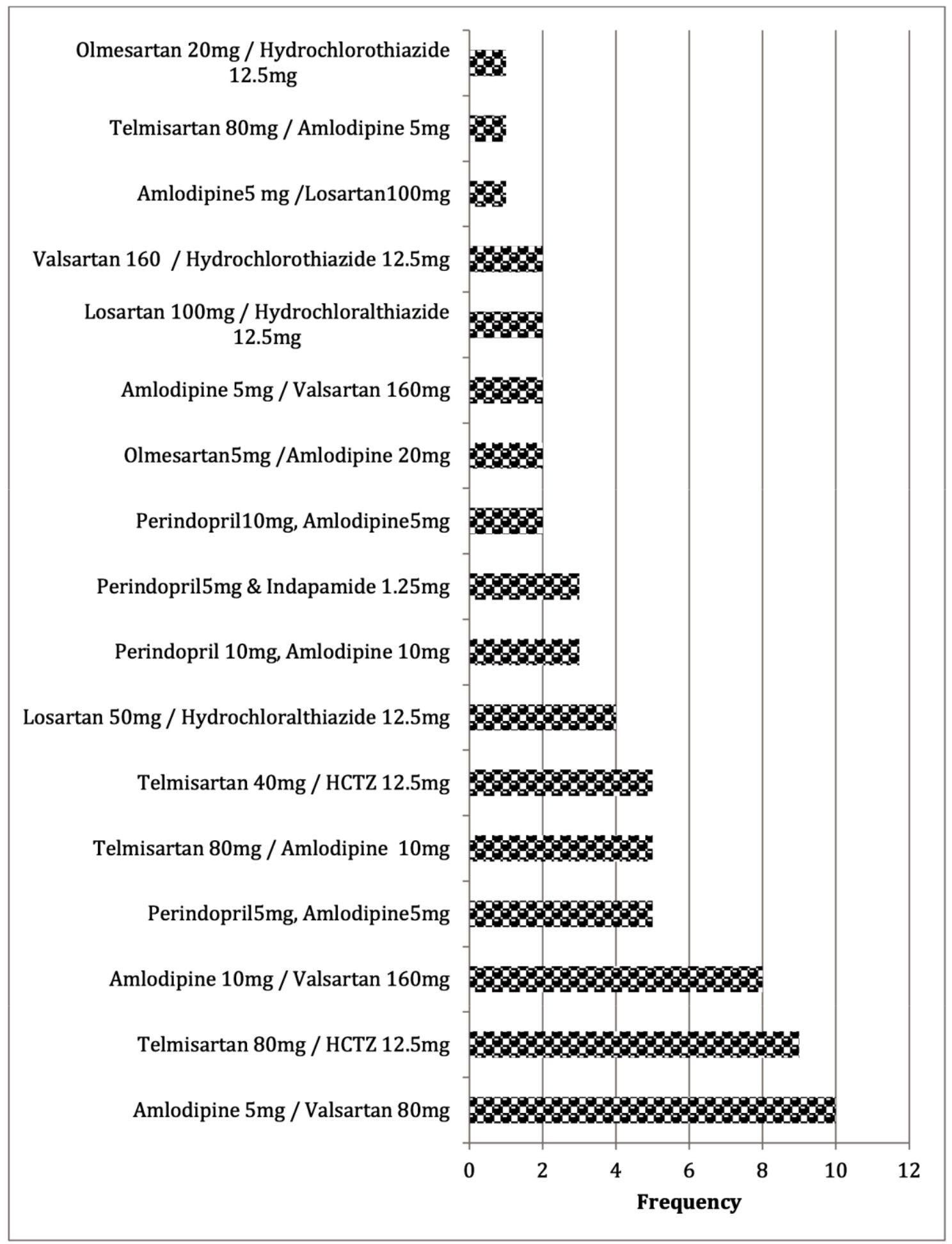

Figure 2. Frequency of two combination drugs prescribed

\section{Defined Daily Dose (DDD) data for monotherapy anti hypertensives prescribed}

DDD/1000 residents/day for single therapy anti-hypertensives

Among the prescriptions selected for this study, $76 \%$ of drugs were prescribed as single drug therapy for the treatment of hypertension. Their dosages per day were estimated to obtain their DDD throughout the year 2017.

The most prescribed antihypertensive drug was proprano- lol 10mg (45 DDD/1000 residents/day). This drug was extensively utilized; other drugs were presented in Fig-ure 3 to compare the utilization. The second most utilized drug was telmisartan $80 \mathrm{mg}$ (0.64 DDD/1000 residents/ day) followed by losartan $50 \mathrm{mg}$ ( $0.61 \mathrm{DDD} / 1000$ resi-dents/day) where both falls under ARB class of drugs.

Amlodipine 10mg (0.03 DDD/1000 residents/day) was the least utilized single therapy anti-hypertensive drug which was utilized almost 20 times lesser compared to telmisartan. 


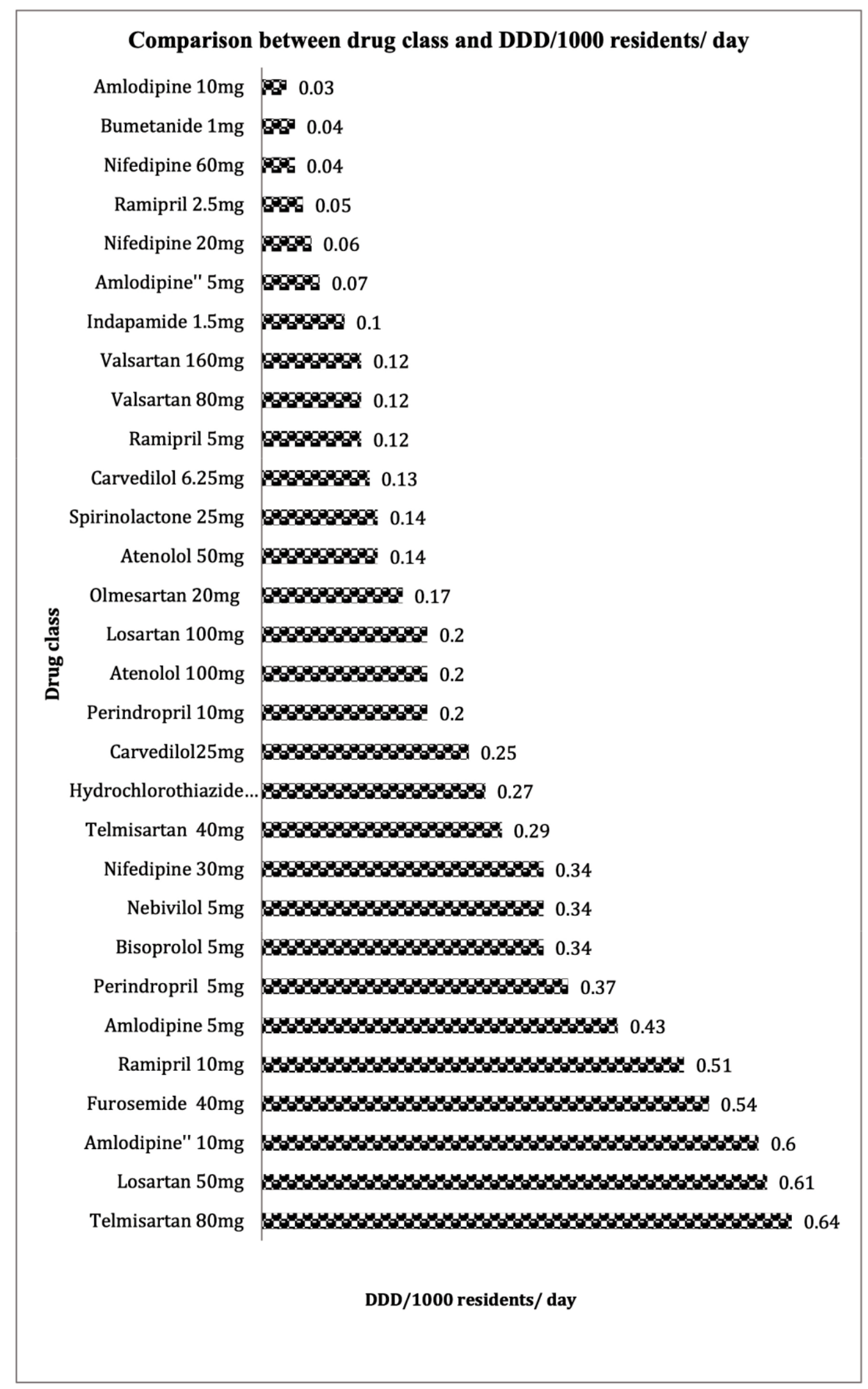

Figure 3. Comparison of drugs according to DDD/1000 residents/day

Analysis of DDD/ user/ day

Figures 4 and 5 shows the comparison between the drugs based on DDD/user/day to determine whether the DDD is close to the average daily maintenance dose as deter-mined by WHODDD. Values nearer to 1 indicates the drugs prescribed for their main indication. Bumetanide
$1 \mathrm{mg}$ is extensively used as it's DDD/user/day is 224 . Other drugs DDD/user/day were compared in the below graph except bumetanide as the value is far different. Nebivilol 5mg and atenolol 100mg $(6.25 \%$ of all 31 single therapy) are the only two drugs that complies to WHO DDD as it has 1DDD/user/day. 


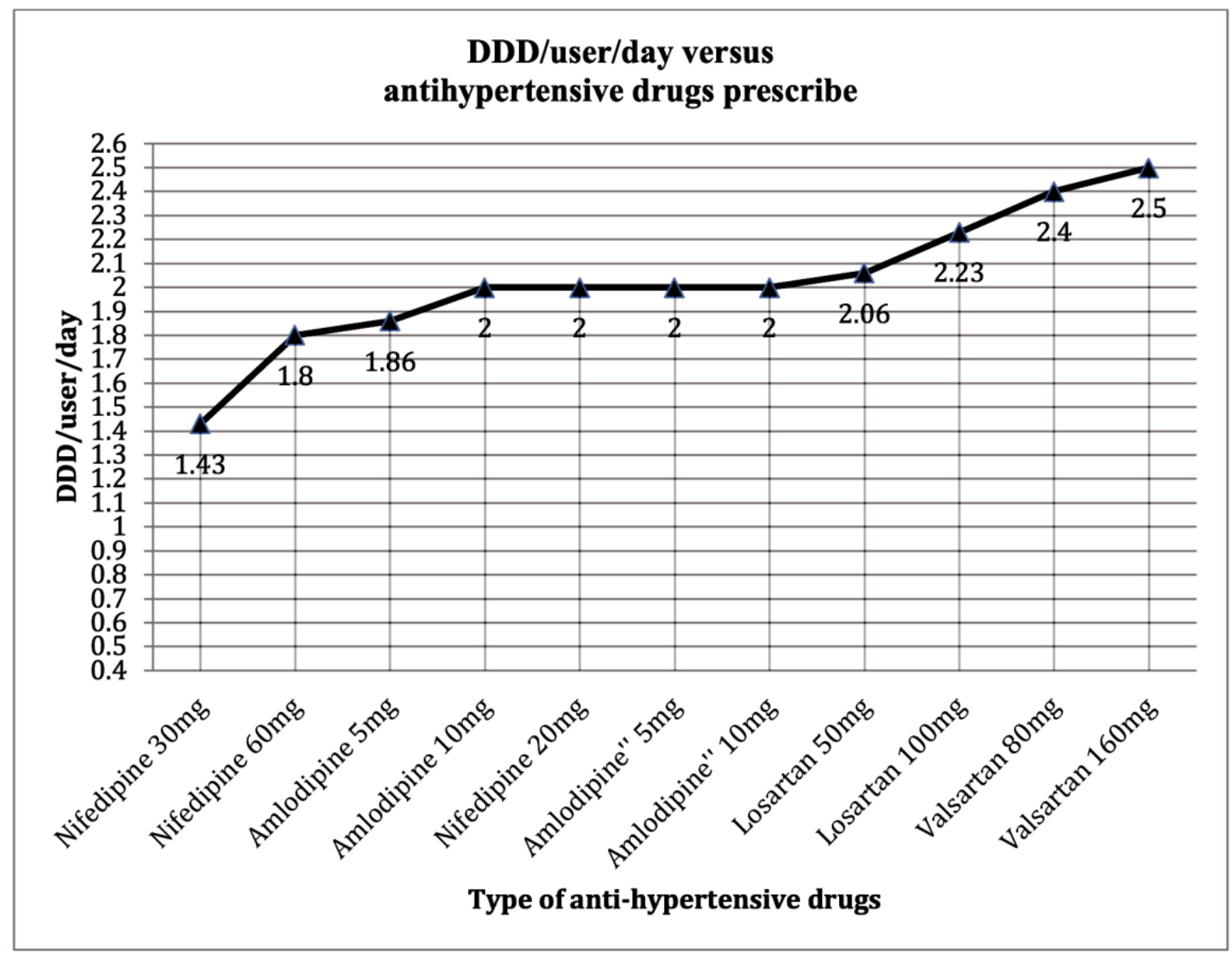

Figure 4. Comparison of drugs according to DDD/day/user

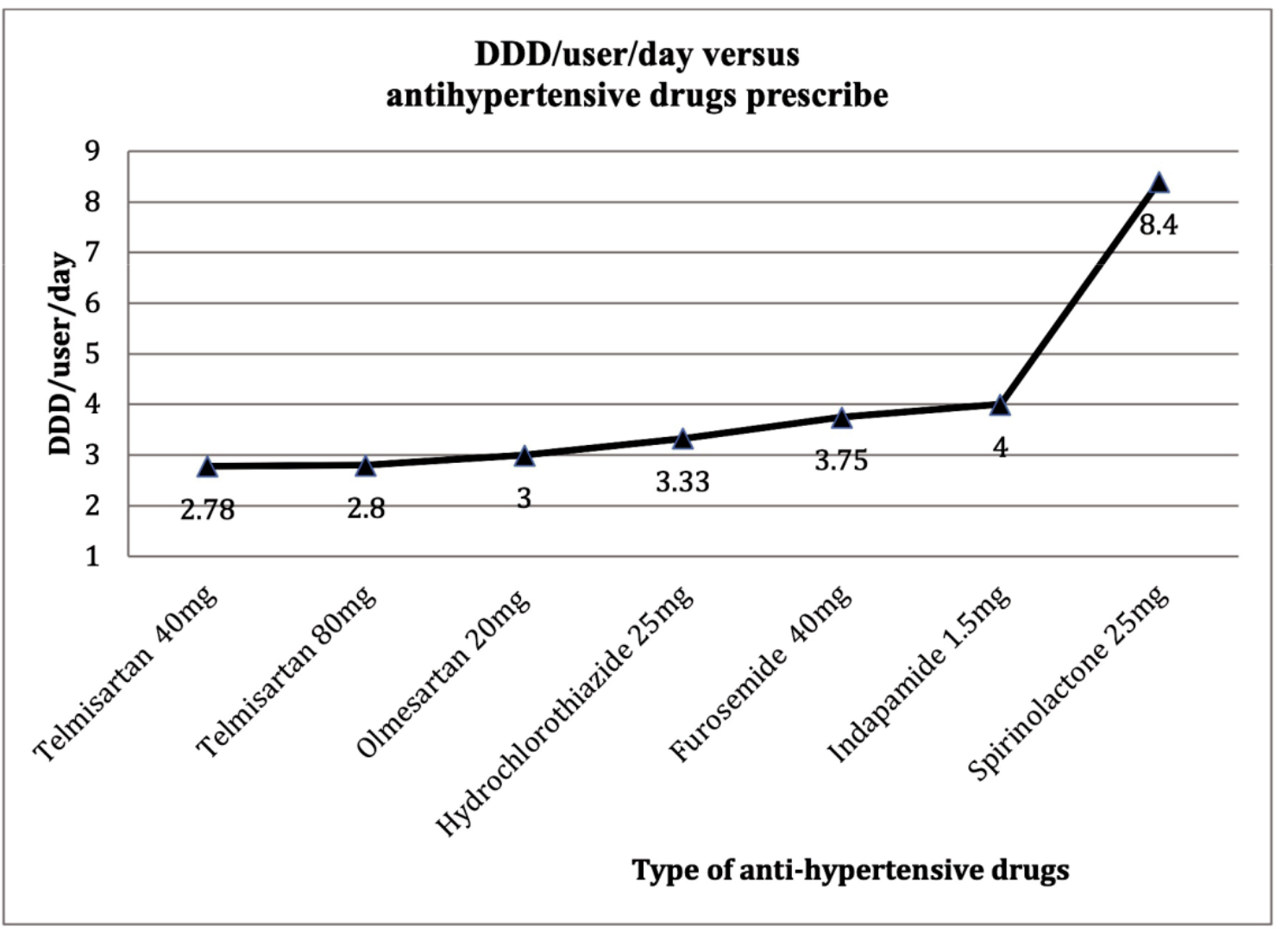

Figure 5. Comparison of drugs according to DDD/day/user 
Defined Daily Dose (DDD) data for fixed dose combination anti hypertensives prescribed

DDD/1000 residents/day for fixed dose combination anti-hypertensives

A total of $24 \%(n=78)$ of the prescribed anti-hypertensive drugs were FDCs. Their dosages per day were es- timated to obtain their DDD throughout the year 2017. All the calculated DDD/1000 residents/day for FDC were lesser than 1; indicating all the FDC drugs (100\%) were underutilized. Among them telmisartan 40mg + HCTZ $12.5 \mathrm{mg}$ were highly used (0.27DDD). The lowest was 3 combination drugs consisting of amlodipine10mg + val-sartan 160mg + HCTZ 25mg (Figure 6).

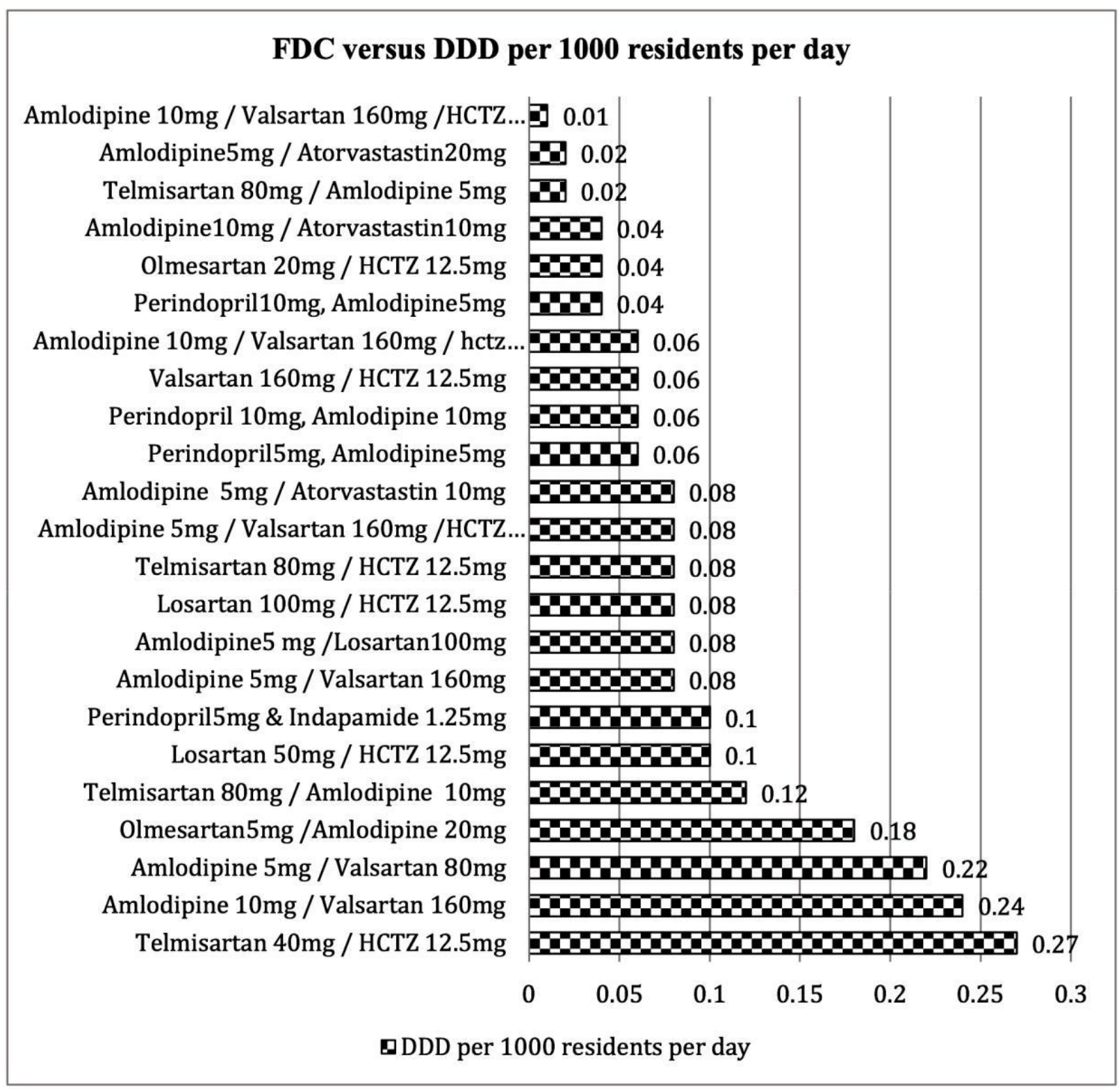

Figure 6. DDD/1000 residents/day comparison of FDC products prescribed

DDD/user/day for fixed dose combination antihyperten-sives

A total of $26 \%(n=6)$ of the FDC drugs has 1 DDD complying to WHO DDD guidelines whereas $65 \%(n=15)$ of the prescribed FDC did not comply with the WHO

DDD. Meanwhile, DDD of $86 \%(n=20)$ of FDC drugs are around the range of 0.76-2. Among these, olmesartan $5 \mathrm{mg}+$ amlodipine $20 \mathrm{mg}$ was the over utilized drug (4.5 DDD) (Figure 4.21-4.22).

\section{Cost of Drug versus Drug Utilization Trend}

Utilization pattern of drugs based on cost factor was shown in Figure 7. Amlodipine 5mg + Atorvastastin 10mg is the drug with the highest cost, RM 6.05. The highly utilized drug is propranolol but it is the cheapest drug among BBs. The second highly utilized drug was amlodipine10mg, apparently it was the highest cost drug among CCB class. Bumetanide $1 \mathrm{mg}$ was the least utilized drug and the cost of the drug was the 3 rd cheapest drugs among all. 


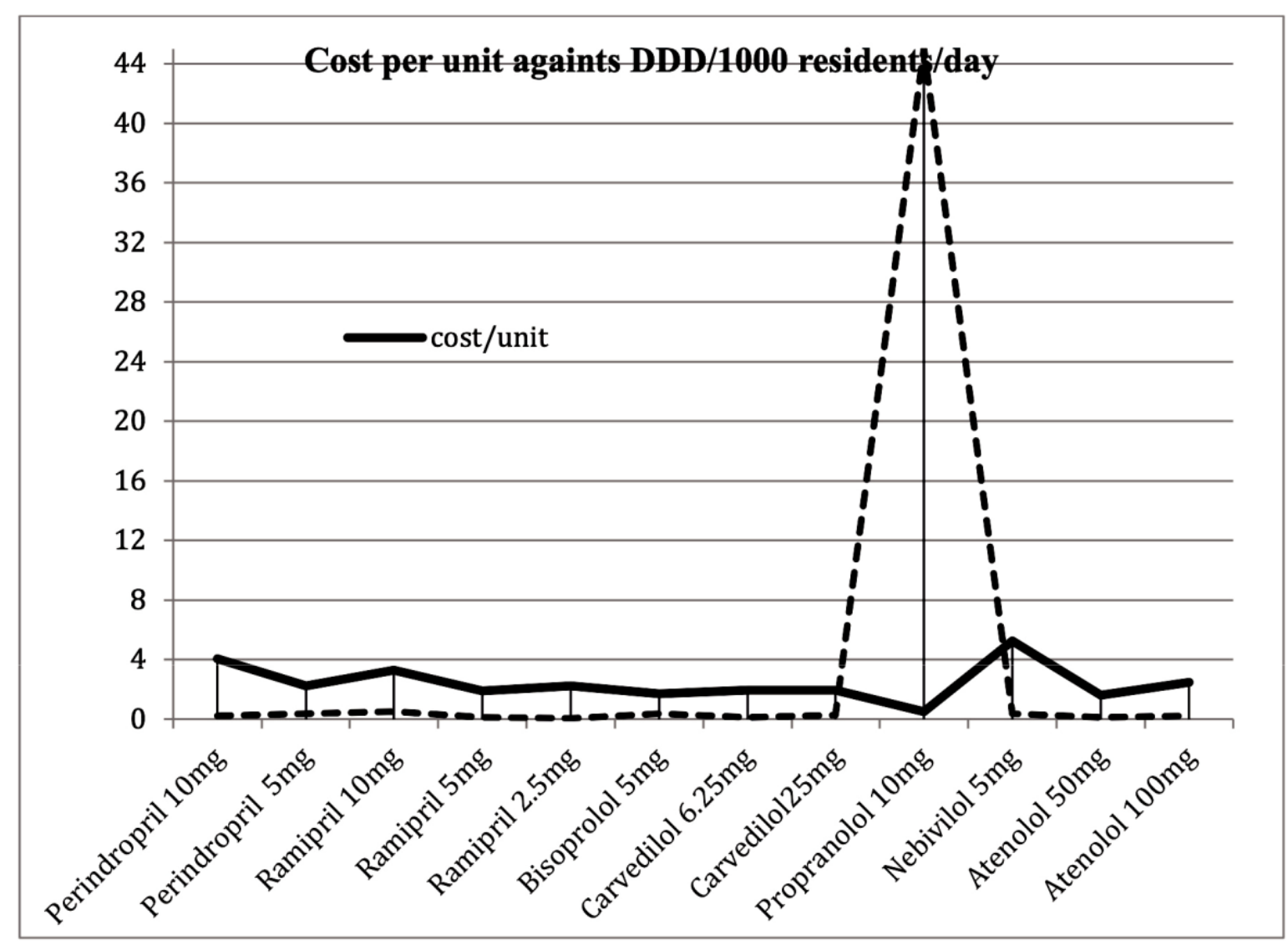

Figure 7. Comparison of DDD/1000 residents/day with cost per unit drug

\section{Discussion}

\section{Sociodemographic Characteristics of Samples}

The results from 12 months study period were based on the data obtained from 200 prescriptions. The sociodemographic characteristics of samples were screened and analyzed based on several variables which include gender, ethnicity and age group ${ }^{[24]}$. Based on the screened prescriptions, percentage of male patients were higher $(52.5 \%)$ compared to females $(47.5 \%)$. In clinical studies, the term 'gender' is used to review basic science studies based on phenotype ${ }^{[25]}$. Previous studies examined the prevalence of hypertension based on gender differences and found women are less likely to have hypertension compared to $\operatorname{men}^{[26]}$. Gender differences could be due to biological and environmental factors. Biological factors including sex hormones, chromosomal differences are being protective for women against hypertension. These become evident during puberty and persistence until reaching menopause at a point where gender differences becomes correspond-ingly smaller $^{[27-29]}$. However, on the contrary, other study has reported females has higher incidence of hyperten-sion than males $^{[30]}$.

Based on ethnicity in this study, Malays (55\%) showed highest percentage of patients receiving antihypertensive therapy followed by Chinese (34\%) and the least is Indians and other races with equal percentages of $5.5 \%$. These findings support previous literature with respect to race/ ethnicity and hypertension in Malaysia suggesting Malays has highest percentage followed by Chinese ${ }^{[24]}$. This may be attributed to the fact that the majority of the local population is Malay.

The study population was sorted into 3 age groups, young adults (ages 18-35), middle-aged adults (ages 36-55), and older adults (aged older than 55 years) ${ }^{[31]}$. The mean age of this study population is 57.81 year-old. The results indicate that older adults are the highest percentage with hypertension, which is more than half of the included samples. Findings of current study also confirm the previous reports ${ }^{[32,33]}$ that risk of hypertension is higher in older women than men. Possibly because low level of estrogen in older women, thus rendering no cardiac protective effect exerted by estrogen. Some age related complications and facing stress may also contribute to the risk of hypertension, such as losing loved ones or even jobs $^{[34]}$.

\section{Prescription analysis}

In this retrospective study, number of drugs per prescription was linked with the indicators such as rational use of drugs and age groups. This study indicated that as the age increases; the number of drugs per prescription also increases. Among the older adults in this study, higher number of people used less than 5 drugs $(24 \%)$. It must be noted that the number of tablets might be misleading because often the fixed dose combination (FDC) drugs 
of anti-hypertensive are counted as one drug. The FDC given is useful to reduce pill load and increase medication adherence. This finding is supported by the previous studies revealing older adults often required multiple drugs to treat multiple health related issues ${ }^{[35]}$.

Results clearly showed that the number of antihypertensive drugs per prescription increased as population ages. Another two studies revealed that elderly hypertensive patients took highest number of drugs ${ }^{[36,37]}$. Several intervention trials show average of two to four antihypertensive drugs may require to achieve effective target of BP control $^{[38,39]}$.

Prescribing indicator like number of drugs per prescrip-tion was used to estimate the degree of polypharmacy or as a determinant of rational use of medicines ${ }^{[40]}$. A total of $30.5 \%$ prescription screened in this study had more than 5 drugs per prescription. The average drug per prescription is 3.89 which is lower than the five drugs benchmark for classification as a polypharmacy event. Generally, use of multiple drugs, especially above five could increase the risk among older patients ${ }^{[41]}$. Receiving more number of drugs increases risk of side effects and interactions. The commonly reported consequences of risk are occurrence of ADR, increased drug cost and decreased patient's compliance.

\section{Drug Utilization Pattern}

The result has shown the most frequently prescribed antihypertensive medications in the corresponding hospital setting were beta blockers $(37 \%)$ followed by diuretics $(23 \%)$ though diuretics are the first line treatment for the management of hypertension according to the JNC VIII guideline. Two studies conducted in India reported BB has the highest percentage $46.7 \%$ and $51 \%$ respectively ${ }^{[42,43]}$. Evidently, the utilization of BB is always greater than diuretics $^{[44]}$. The evidence of using $\mathrm{BB}$ is strong in hypertensive patients with heart failure, acute coronary syndromes, and arrhythmias.

ACE inhibitors are the lowest (9\%) used drug in our study which in congruent with the findings reported at Panjab University Health Centre in India, with usage of 3.9\%[30]. Another study recorded highest usage of ACEi (59\%) unlike current study. This may be because sample in that study was with hypertension and diabetes mellitus ${ }^{[30]}$.

Bisoprolol is the drug prescribed to most samples in current study. Bisoprolol is the drug of choice for hypertensive patients with certain other cardiac diseases and also for elderly. Bisoprolol efficient in once daily dosing and provides well tolerability in elderly ${ }^{[45,46]}$. Highlighting that our study mostly consists of elderly patients whom have higher chances to be with comorbidities because of high drugs per prescription were seen as aging.

Some studies proved that combination therapies are effective to lower the $\mathrm{BP}^{[47]}$. In current study, it is found that combination therapy of $\mathrm{CCB}$ and $\mathrm{ARB}$ were highly used. Evidently, low-dose combination therapy with CCBs and ARBs was found to give effective BP con- trol than using high-dose monotherapy ${ }^{[48]}$. Further evidence showed by Kuschnir, Bendersky ${ }^{[49]}$, combination of low-dose nifedipine with losartan was associated with improved BP control than monotherapy $(\mathrm{p}<0.05)$ in patients with mild-to-moderate hypertension.

The combination drugs that highly used in this study was Amlodipine + Valsartan. Similarly, the share of CCB mostly occurs in few studies ${ }^{[14]}$. FDC drugs $(22 \%)$ were used less than single dose therapy in this study. Higher use of monotherapy in relation to combination drug therapy is similar to studies done in India ${ }^{[30,42]}$ and Ethiopia ${ }^{[50]}$. The finding is however different in studies done in Chandigarh $^{[43]}$ where less patients received monotherapy than combination therapy. The differences could be due to patient condition like the severity of their disease and prescribing pattern depends on prescriber's choice too.

\section{Drug utilization based on DDD calculation for antihypertensive prescribed}

The DDD is a statistical measure to identify drug consumption. By evaluating the DDD of the drugs, the overview on anti-hypertensive utilization can be attained.

DDD per 1000 residents per day was calculated and compared. It provides a rough estimate of the proportion of the population treated daily with a specific drug. The high value DDD indicating mostly utilized.

The most utilized anti-hypertensive drugs are telmisartan and losartan as single drugs. For FDC combination of telmisartan and hydrochlorothiazide was utilised the most. Other researchers concluded the use of ARB in hypertensive are more in hypertensive patients with diabetics ${ }^{[42,51]}$. Hydrochlorothiazide acts by inhibiting $\mathrm{Na}+\mathrm{Cl}-$ co-transporter while losartan blocks angiotensin II receptor. Various studies suggest volume reliant status of blood pressure in salt encumbered patients can be changed to a favorable renin reliant status by addition of diuretic, since ACEIs and ARBs are less effective in the former situation ${ }^{[52]}$. The Joint National Committee (JNC VII) guidelines recom-mend thiazide diuretics to be prescribed alone or as part of combination therapy for most hypertensive patients with-out compelling indications ${ }^{[53]}$.

DDD rates per user per day are calculated to determine whether the DDD is close to the average maintenance dose for the drugs main indication (as determined by WHO). This measure is expected to be close to 1 for drugs used for chronic diseases.

Only $14 \%$ of the overall type of drugs presented, complies with WHO DDD guideline as they have reached 1 DDD.

DDD is just average maintenance dose; the DDD does not necessarily reflect the recommended dose. Differences may be caused by the severity of diseases or different indications for a drug. Underutilized drugs could be due to prescribed not for main indication or start up doses.

The DDD for $39 \%$ of those drugs more than twice the DDD recommended by WHO. There are other study where the DDD doesn't comply to $\mathrm{WHO}^{[54]}$. One reason for these discrepancies may be that the drug classes under study namely ACEi and BBs could have been 
prescribed for reasons other than hypertension. This explanation seems to be reasonable as represented drugs of choice not only for the treatment of hypertension, but also of heart failure. Although we have no information about the indication for the medication of each individual, it is reasonable to assume that the indication for a prescription does not change when the antihypertensive drug for the individual patient changes.

\section{Cost and drug utilization}

The findings show a direct relationship between the drug utilization and drug cost in all anti-hypertensive drug groups for instance, propranolol is the highly used drug with the lowest cost among BB category. Prescribing trend based of affordability will further increase the drug com-pliance among the patients. Interestingly, this study also support the claim from previous study who reported that medical consultants tend to prescribe newer agents that might have a higher cost due to the innovator status ${ }^{[14]}$.

\section{Conclusion}

The study findings revealed that higher percentage patients were Malays compared to other ethnics and male gender is more than the female in the whole sample. The age group that showed highest percentage was older adults (more than 55 years old) compared to middle aged adults ( $>36$ years to 55 years) and young adults $(>18$ years to 35 years old). Drugs prescribed per prescription among 200 patients ranged from 1 to 6 types of drug products. The mean number of drugs prescribed per prescriptions was 3.89 and standard deviation of 2.37. Beta blockers are the most commonly prescribed group of antihypertensives (37\%). Bisoprolol 5mg (9\%) and Furosemide $40 \mathrm{mg}(8.7 \%)$ were those drugs taken by most patients in this study. Out of 330 anti-hypertensive drug products pre-scribed, $76 \%$ of them were non-FDC drugs. Prescribing trend shifted towards the newer antihypertensive drugs as evidenced by certain FDC products doubled the DDD value recommended by WHO.

\section{Conflict of Interest}

The authors declare that there is no conflict of interest in this work.

\section{Reference}

1. Foex P. Hypertension: pathophysiology and treatment. Contin Educ Anaesth Crit Care \& Pain 2004; 4

2. Beevers G, Lip GYH and O'Brien E. The pathophysiology of hypertension. BMJ 2001; 322: 912-916.

3. Datta S. Utilization Study of Antihypertensives in a South Indian Tertiary Care Teaching Hospital and Adherence to Standard Treatment Guidelines. J Basic Clin Pharm 2016; 8: 33-37. doi: 10.4103/09760105.195100 .

4. Go AS, Mozaffarian D, Roger VL, et al. Heart disease and stroke statistics-2014 update: a report from the American Heart Association. Circ 2014; 129: e28-e292. 2013/12/20. doi: 10.1161/01. cir.0000441139.02102.80

5. Jarari N, Rao N, Peela JR, et al. A review on prescribing patterns of antihypertensive drugs. Clin. Hypertens. 2016; 22: 7. doi: 10.1186/ s40885-016-0042-0.

6. Gupta R and Guptha S. Strategies for initial management of hyperten-sion. Indian J. Med. Res. 2010; 132: 531-542.

7. Whelton PK, Carey RM, Aronow WS, et al. 2017 ACC/AHA/AAPA WBC/ACPM/AGS/APhA/ASH/ASPC/NMA/PCNA Guideline for the Prevention, Detection, Evaluation, and Management of High

Blood Pressure in Adults. A Rep. of the Am. Coll. Cardiol./Am Heart Assoc Task Force on Clin Pract Guidel 2017. doi: 10.1016/j. jacc.2017.11.006

8. Danaei G, Finucane MM, Lin JK, et al. National, regional, and global trends in systolic blood pressure since 1980: systematic analysis of health examination surveys and epidemiological studies with 786 country-years and 5.4 million participants. Lancet 2011 377: 568- 577. 2011/02/08. doi: 10.1016/s0140-6736(10)62036-3.

9. Mills KT, Bundy JD, Kelly TN, et al. Global Disparities of Hyperten-sion Prevalence and Control: A Systematic Analysis of Population-Based Studies From 90 Countries. Circ. 2016; 134: 441450. doi: 10.1161/CIRCULATIONAHA.115.018912.

10. Abas A. One in three adult Malaysians have hypertension. New Strait Times [newspaper on the Internet]. 2016 June 6. Available from: https://www.nst.com.my/news/2016/06/150074/one-three-adult-malaysians-have-hypertension

11. National Health And Morbidity Survey Report no. (NMRR - 14-1064 21877), 2015. Kuala Lumpur: Institute for Public Health, Ministry of Health, Malaysia.

12. Aprahamian I, Sassaki E, Dos Santos MF, et al. Hypertension and frailty in older adults. J Clin Hypertens 2017. doi: 10.1111/jch.13135.

13. Park C, Fang J, Hawkins NA, et al. Comorbidity Status and An-nual Total Medical Expenditures in U.S. Hypertensive Adults. Am J Prev Med 2017; 53: S172-S181. 2017/11/21. doi: 10.1016/j. amepre.2017.07.014.

14. Rachana PR, Anuradha HV, MC Shivamurthy. Anti hypertensive pre-scribing patterns and cost analysis for primary hypertension: a retro-spective study. J Clin Diagn Res 2014; 8: HC19-22. doi: 10.7860/ JCDR/2014/9567.4890.

15. Pharmaceutical Services Programme, Ministry of Health Malaysia. Malaysian Statistics on Medicines 2011-2014. Published 2017.

16. Hussain IM, Naqvi BS, Qasim RM, et al. Current trends in treatment of hypertension in Karachi and cost minimization possibilities. Pak J Med Sci 2015; 31: 1021-1026.

17. Shalini S, Ravichandran V, Dhanaraj SK, et al. Drug Ulitization Stud-ies - An overview. Int. J. Pharm. Sci. Nanotechnol. 2010; 3: 8.

18. Soos G. Importance of drug utilization studies as a pharmaceutical tool for the practice of evidence-based medicine. Acta. Pharm. Hung. 2002; 72: 252-256.

19. KPJ Healthcare Berhad. Annual Report 2015.

20. Devane D, Begley CM and Clarke M. How many do I need? Basic principles of sample size estimation. J Adv Nurs 2004; 47: 297-302. 2004/07/09. doi: 10.1111/j.1365-2648.2004.03093.x.

21. Nanjwade BK. Study on drug utilization pattern of antihypertensive agents in a tertiary care hospital. World J. Pharm. Pharm. Sci. 2016; 5: 1078-1088.

22. Truter I. Ethical issues related to retrospective drug utilization studies in South Africa. Pharmacoepidemiol Drug Saf 2001; 10:679_ 683. doi: $10.1002 /$ pds. 647 .

23. Evans JM, McNaughton D, Donnan PT, et al. Pharmacoepidemiologi-cal research at the Medicines Monitoring Unit, Scotland: Data pro-tection and confidentiality. Pharmacoepidemiol Drug Saf 2001; 10: 669-673. doi: $10.1002 /$ pds.644.

24. Abdul-Razak S, Daher AM, Ramli AS, et al. Prevalence, awareness, treatment, control and socio demographic determinants of hypertension in Malaysian adults. BMC Public Health 2016; 16.

25. Gillis EE and Sullivan JC. Sex Differences in Hypertension: Recent Advances. Hypertens 2016; 68: 1322-1327.

26. Everett B and Zajacova A. Gender Differences in Hypertension and Hypertension Awareness Among Young Adults. Biodemography Soc. Biol. 2015; 61: 1-17.

27. Sandberg K and Ji H. Sex differences in primary hypertension. Biol Sex Differ 2012; 3: 7. doi: 10.1186/2042-6410-3-7.

28. Vitale C, Fini M, Speziale G, et al. Gender differences in the cardiovascular effects of sex hormones. Fundam Clin Pharmacol 2010; 24 675-685. doi: 10.1111/j.1472-8206.2010.00817.x.

29. Vitale C, Mendelsohn ME and Rosano GM. Gender differences in the cardiovascular effect of sex hormones. Nat. Rev. Cardiol. 2009; 6: 532-542. doi: 10.1038/nrcardio.2009.105.

30. Tiwari H, Kumar A and Kulkarni SK. Prescription monitoring of antihypertensive drug utilisation at the Panjab University Health Centre in India. Singapore Med J 2004; 45: 117-120.

31. Petry NM. A comparison of young, middle-aged, and older adult treatment-seeking pathological gamblers. The Gerontologist 2002; 42: 92-99.

32. Mozaffarian D, Benjamin EJ, Go AS, et al. Heart disease and stroke statistics-2015 update: a report from the American Heart Association. Circ. 2015; 131: e29-322. doi: 10.1161/cir.0000000000000152.

33. van Rossum CT, van de Mheen H, Witteman JC, et al. Prevalence, treatment, and control of hypertension by sociodemographic factors among the Dutch elderly. Hypertens. 2000; 35: 814-821.

34. Eshkoor SA, Hamid TA, Shahar S, et al. Factors Affecting Hypertension among the Malaysian Elderly. J. Cardiovasc. Dev. Dis. 2016; 3. 
35. Willcox SM, Himmelstein DU and Woolhandler S. Inappropriate drug prescribing for the community-dwelling elderly. JAMA 1994; 272: 292-296

36. Kroenke K and Pinholt EM. Reducing polypharmacy in the elderly. A controlled trial of physician feedback. J. Am. Geriatr. Soc. 1990; 38: $31-36$.

37. Stewart RB and Cooper JW. Polypharmacy in the aged. Practical solutions. Drugs Aging 1994; 4: 449-461.

38. UK Prospective Diabetes Study Group. Tight blood pressure control and risk of macrovascular and microvascular complications in type 2 diabetes: UKPDS 38. BMJ 1998; 317: 703-713.

39. Agodoa LY, Appel L, Bakris GL, et al. Effect of ramipril vs amlodipine on renal outcomes in hypertensive nephrosclerosis: A randomized controlled trial. JAMA 2001; 285: 2719-2728.

40. Desalegn AA. Assessment of drug use pattern using WHO prescrib-ing indicators at Hawassa University teaching and referral hospi-tal, south Ethiopia: a cross-sectional study. BMC Health Serv. Res. 2013; 13: 170. doi: 10.1186/1472-6963-13-170.

41. Goh CR. Minimising polypharmacy - a challenge in palliative care. Singapore Med J 2002; 43: 273-275.

42. Jhaj R, Goel NK, Gautam CS, et al. Prescribing patterns and cost of antihypertensive drugs in an internal medicine clinic. Indian Heart $\mathbf{J}$ 2001; 53: 323-327.

43. Malhotra S, Karan RS, Pandhi P, et al. Pattern of use and pharmaco-economic impact of antihypertensive drugs in a north Indian referral hospital. Eur J Clin Pharmacol 2001; 57: 535-540.

44. James PA, Oparil S, Carter BL, et al. 2014 evidence-based guideline for the management of high blood pressure in adults: report from the panel members appointed to the Eighth Joint National Committee (JNC 8). JAMA 2014; 311: 507-520. doi: 10.1001/ jama.2013.284427.

45. Broncel $\mathrm{M}$, Chojnowska-Jezierska J and Adamska-Dyniewska $\mathrm{H}$. Bisoprolol in the treatment of hypertension in the elderly. J Hum Hypertens 1998; 12: 643-644.

46. Weiner L and Frithz G. Antihypertensive effects of bisoprolol during once daily administration in patients with essential hypertension. A dose-ranging study with parallel groups. Eur J Clin Pharmacol 1986; 29: 517-521.

47. Brenner BM, Cooper ME, de Zeeuw D, et al. Effects of losartan on renal and cardiovascular outcomes in patients with type 2 diabetes and nephropathy. N Engl J Med 2001; 345: 861-869. doi: 10.1056/ NEJMoa011161.

48. Andreadis EA, Tsourous GI, Marakomichelakis GE, et al. High-dose monotherapy vs low-dose combination therapy of calcium chan-nel blockers and angiotensin receptor blockers in mild to moderate hypertension. J Hum Hypertens 2005; 19: 491-496. doi: 10.1038/ sj.jhh. 1001843

49. Kuschnir E, Bendersky M, Resk J, et al. Effects of the combination of low-dose nifedipine GITS $20 \mathrm{mg}$ and losartan $50 \mathrm{mg}$ in patients with mild to moderate hypertension. J Cardiovasc Pharmacol 2004; 43: 300-305.

50. Shukrala F and Gabriel T. Assessment of prescribing, dispensing, and patient use pattern of antihypertensive drugs for patients attending outpatient department of Hiwot Fana Specialized University Hospital, Harar, Eastern Ethiopia. Drug Des Devel Ther 2015; 9: 519-523. doi: $10.2147 /$ dddt.s73670.

51. Dhanaraj E, Raval A, Yadav R, et al. Prescription Pattern of Antihypertensive Agents in T2DM Patients Visiting Tertiary Care Centre in North India. Int J Hypertens 2012; 2012: 520915. doi: 10.1155/2012/520915.

52. Busari OA, Oluyombo R, Fasae AJ, et al. Prescribing Pattern and Utilization of Antihypertensive Drugs and Blood Pressure Control in Adult Patients with Systemic Hypertension in a Rural Tertiary Hospi-tal in Nigeria. Am J Intern Med 2014; 2(6): 144-149.

53. Chobanian AV, Bakris GL, Black HR, et al. Seventh report of the Joint National Committee on Prevention, Detection, Evaluation, and Treat-ment of High Blood Pressure. Hypertens. 2003; 42: 12061252. doi: 10.1161/01.HYP.0000107251.49515.c2.

54. Grimmsmann $\mathrm{T}$ and Himmel W. Discrepancies between prescribed and defined daily doses: a matter of patients or drug classes? Eur J Clin Pharmacol 2011; 67: 847-854. doi: 10.1007/s00228-011-1014-7. 